\title{
Positive serum specific lgE has a short half-life in patients with penicillin allergy and reversal does not always indicate tolerance
}

\author{
Janni Hjortlund ${ }^{1 *}$, Charlotte Gotthard Mortz ${ }^{1}$, Tore Bjerregaard Stage ${ }^{2}$, Per Stahl Skov ${ }^{1}$, Ronald Dahl ${ }^{1}$ \\ and Carsten Bindslev-Jensen ${ }^{1}$
}

\begin{abstract}
Background: The positive and negative predictive values of specific lgE to penicillins are not well established for penicillin hypersensitivity. One reason may be that serum IgE levels to penicillin diminish over time. The objective in this study was to investigate variations in serum half-life (T1/2) for specific lgE to penicillins (s-lgE) and to evaluate the outcome of penicillin challenges in patients with previous but not present specific lgE to penicillins.

Methods: Two subgroups were investigated. All included patients had a history of penicillin allergy with reported symptoms such as urticaria/angioedema or unclassified cutaneous rash. T1/2 of specific IgE to penicillins was calculated based on sera from 29 patients with repeated measurements of s-lgE. Twenty-two patients with a previous positive s-lgE was followed and challenged with penicillin when IgE had become negative.

Results: The T1/2 for s-lgE varied between the 26 patients with decreasing s-lgE from 1.6 months to 76.4 months and $52 \%$ had a $T 1 / 2$ of less than a year. The three patients with stable and increasing IgE-values showed $T 1 / 2$ approaching infinity A total of 29 challenges with $\beta$-lactams were performed. Four different patterns were seen when evaluating the clinical reaction to challenge (positive/negative) and post-challenge boost of s-lgE (yes/no). Eight (36.4\%) had negative challenge and negative post-challenge s-lgE, eight (36.4\%) negative challenge, but positive post-challenge s-lgE levels. $3(13.6 \%)$ had positive challenge and positive post-challenge s-IgE whereas 3 (13.6\%) were challenge positive, but had negative post-challenge s-lgE.
\end{abstract}

Conclusion: Specific IgE to penicillins declines over time stressing the importance of a close time relation between diagnostic work-up and clinical reaction. Reversal of previously positive s-IgE may still be associated with positive penicillin challenges and/or re-boostering of s-lgE to positivity.

Keywords: Penicillin allergy, 7-day oral challenge, Prolonged challenge, Specific IgE to penicillin, Half-life

\section{Background}

Correct diagnosis of allergy to penicillin is of great importance, but in contrast to what is found in a patient with a history of grass pollen allergy, discrepancy between skin test, measurement of specific IgE and outcome of challenge is often seen in patients with suspected penicillin allergy. Recommendations to patients based on falsenegative results can lead to severe adverse reactions whereas false-positive results can lead to administration of

\footnotetext{
* Correspondence: janni.hjortlund@rsyd.dk

'Department of Dermatology and Allergy Centre, Odense University Hospital, Sdr. Boulevard 29, DK-5000 Odense C, Denmark

Full list of author information is available at the end of the article
}

less effective and more expensive drugs. We have previously demonstrated $[1,2]$ that challenge tests with penicillin play an important role in the diagnosis of hypersensitivity reactions to penicillin. Including a 7-day treatment with a therapeutic dose of penicillin is of special importance because patients with negative skin test and negative specific IgE to penicillins, but with a convincing history of penicillin allergy, may be positive in such a prolonged challenge test with therapeutic dosing for 7 days. International guidelines (ENDA and ICON) [3-6] only propose a single dose challenge. The positive and negative predictive values of serum specific IgE to penicillins (s-IgE) are not well established [7-9]. One reason may be that s-IgE levels to 
penicillin diminish over time [9-12]. One of the aims of this study was to determine the serum half-life $\left(\mathrm{T}^{1 / 2}\right)$ of s-IgE. The second aim was to assess the clinical importance of previous IgE sensitization in patients, where s-IgE (penicillin V and G, ampicillin and amoxicillin) had become negative. To our knowledge a study on penicillin challenges has never been performed in patients with previous, but not present measurable values of specific IgE to penicillins.

\section{Methods \\ Patients}

In the period September 2003 to October 2013, our serum bank contains samples from 2791 adult patients with suspected penicillin allergy, who had performed measurements of $\mathrm{s}$-IgE to penicillins. Hereof a total of 287 adult patients had a positive s-IgE to one or more penicillins (benzylpenicillin (pen G), phenoxymethylpenicillin (pen V), ampicillin (AMP), amoxicillin (AX)).

Serum elimination half-life ( $\left.T^{1} / 2\right)$ of $s$-IgE (pen $G, V, A M P, A X$ ) A total of 29 patients ( 10 men and 19 women) had 3 or more measurements of $\mathrm{s}$-IgE over a period of up to 59 months. These were included for determination of $\mathrm{T} 1 \frac{1}{2}$ of s-IgE (pen G, V, AMP, AX), Figure 1. The median level of s-IgE measurements was 3 measurements (range 3 - 15 measurements). There were no demographic differences between the patients included and the patients with less than three IgE-measurements not included. Further no demographic differences were found between the patients with positive $\mathrm{s}-\mathrm{IgE}$ and the patients with negative $s-\operatorname{IgE}(p>0.05)$. The most frequently reported symptoms were urticaria and/or angioedema (17 cases, $58.6 \%$ ), unclassified cutaneous rash (11 cases, 37.9\%) and anaphylaxis (1 case, $3.5 \%$ ). Pen V was the culprit drug in most cases, 21 (72.4\%), the remaining reacting to dicloxacillin (DX) (4 cases, 13.8\%), AX (3 cases, 10.3\%) and unknown in one case $(3.5 \%)$.

\section{Penicillin challenge in patients with previous positive specific IgE to penicillins}

The serum bank contained sera from forty-two patients followed prospectively with repeated measurements of $\mathrm{s}$-IgE that had reverted to negative test results. Of these twenty-two (12 men and 10 women) agreed to participate in the present study. All patients had negative skin prick test (SPT) and intracutaneous test (ICT) to penicillins prior to penicillin challenge, Figure 1.

The $\beta$-lactam eliciting the initial reaction (culprit drug) was pen $\mathrm{V}$ in 15 cases (62.5\%), AX in 5 (20.8\%) and DX in $2(8.3 \%)$.

According to the history reactions commencing within $1 \mathrm{~h}$ after drug intake were classified as immediate reactions (IR) and occurred in 7 patients. Reactions commencing $1 \mathrm{~h}$ or longer after drug intake were classified as nonimmediate reactions (NIR) and seen in 15 patients. Twelve of the 22 patients reported urticaria and/or angioedema (6 IR, 6 NIR) and 10 patients with unclassified cutaneous rash (1 IR, 9 NIR). Sixteen of the 22 patients had experienced their initial clinical reaction within one year prior to measurement of the positive s-IgE (thirteen even within 6 months), two within 1-5 years and 4 more than five years prior to blood sampling.

All patients showed undetectable levels of s-IgE at the time of penicillin challenge. The challenge tests were performed according to procedures in our two previous studies $[1,2]$, with the modifications described in Figure $2 \mathrm{a}+$ $2 \mathrm{~b}$. At visit 1 , a titrated challenge with penicillin was performed by intravenous injection of pen $G$ from 100 IE to 1.000.000 IE in 10-fold increments and $20 \mathrm{~min}$ interval,

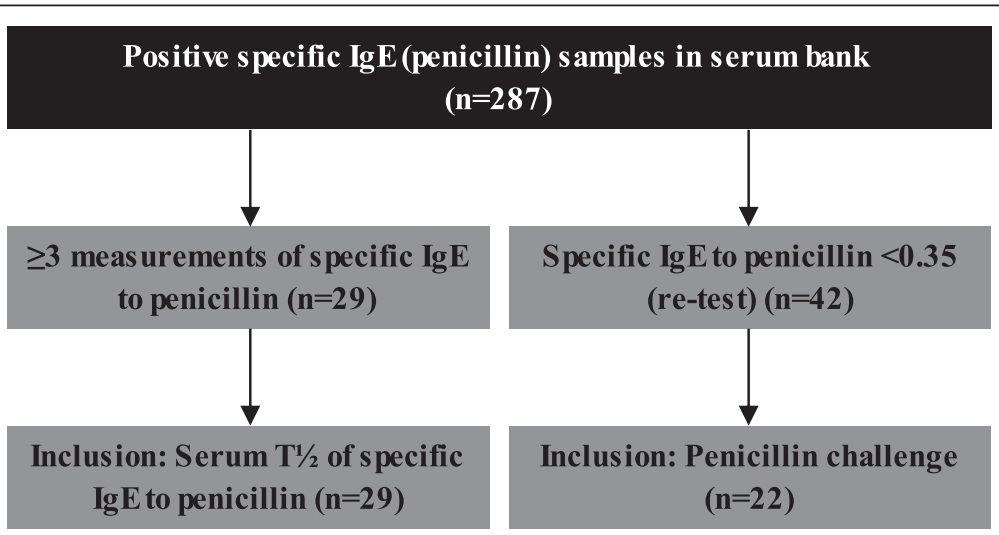

Figure 1 Algorithm for inclusion criteria. 29 patients included for the estimation of $T \frac{1}{2}$ of specific lgE to penicillins. 22 patients included in penicillin challenge in patients with previous lgE sensitization to penicillins. A total of 50 individuals were included, since 3 subjects were included in both the penicillin challenge in patients with previous IgE sensitization to penicillin and in the estimation of $T 1 / 2$ of specific IgE to penicillins. IgE: Immunoglobulin E, T1/2: half-life for specific IgE to penicillins. 
$\mathbf{a}$

Challenges

\begin{tabular}{llll} 
Visit 1 & \multicolumn{1}{c}{ Visit 2 } & Visit 3 & Visit 4 \\
\hline & & & Culprit drug challenge \\
$\begin{array}{l}\text { Pen G and V } \\
\text { challenge }\end{array}$ & $\begin{array}{l}\text { Pen V challenge } \\
\text { 7-day oral challenge } \\
\text { (p.o.7) with therapeutic } \\
\text { doses }\end{array}$ & $\begin{array}{l}\text { Culprit drug } \\
\text { challenge } \\
\text { (Titrated single } \\
\text { dose challenge) }\end{array}$ & $\begin{array}{l}\text { 7-day oral challenge } \\
\text { (p.o.7) with therapeutic } \\
\text { doses }\end{array}$
\end{tabular}

b

Specific IgE measurements

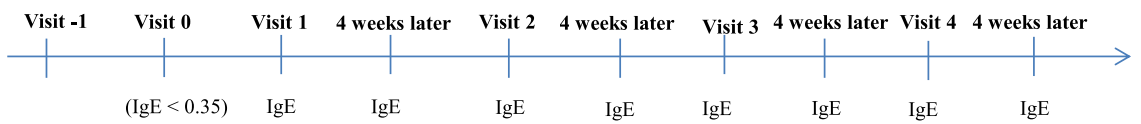

Figure $\mathbf{2}$ Challenge program in the patients with previous positive specific lgE to penicillins. (A) Challenge program within each visit. (B) Program for measurements of specific lgE to penicillins. In continuation of each challenge, specific IgE measurements were performed and repeated 4 weeks later. In case of any positive clinical reaction or renewed positive specific lgE, further challenges were terminated. Visit -1: The visit of the first positive measurement of specific IgE to penicillin. Visit 0: The visit of the first negative measurement of specific IgE to penicillin. Pen G: Benzylpenicillin, Pen V: Phenoxymethylpenicillin, p.o.7: prolonged oral challenge with penicillin for 7 days, IgE: specific immunoglobulin E to penicillins (Pen G, Pen V, ampicillin, amoxicillin). All patients had negative specific IgE to penicillin at the time of penicillin challenge (visit 0 and 1 ).

followed by administration of a single oral dose of $400 \mathrm{mg}$ pen V 20 min after the last i.v. dose. If negative, the patient was at visit 2 given the first two doses $(80 \mathrm{mg}$ followed by $800 \mathrm{mg} 30 \mathrm{~min}$ later) of a 7-day course of pen $\mathrm{V}$ in the clinic. If this challenge was negative, the patient continued with $800 \mathrm{mg}$ t.i.d. at home. If the above challenges with pen $\mathrm{G}$ and $\mathrm{V}$ proved negative and if the case history or previous positive specific IgE pointed to a reaction to a different $\beta$-lactam antibiotic, the patient was subsequently challenged with the culprit drug (AX, AMP, DX) in a titrated oral protocol (visit 3). Three doses were administered with 30-min. interval (5, 50 and $500 \mathrm{mg})$. If negative, the patient was at visit 4 given the first dose of a 7-day course of culprit drug $(500 \mathrm{mg})$ followed by dosing (500 mg b.i.d. (AMP, AX)), and (500 mg t.i.d. (DX)) at home. In case of a positive reaction (positive challenge or positive s-IgE) further challenges were terminated. Blood samples for determination of s-IgE were drawn at visit -1 , $0,1,2,3,4$ and four weeks after each visit. In case of renewed positive s-IgE after any of the visits, the investigation was stopped and the patient was followed with measurement of s-IgE with 3-6 months interval.

The Regional Scientific Ethical Committees for Southern Denmark has approved the study (approval number S-20100091). All patients gave written informed consent for the study as well as consenting to storage of their blood in the serum bank.

\section{Measurement of specific $\lg E$ to penicillins (s- $\lg E$ )}

S-IgE levels to pen G, pen V, AMP, AX and MDM (minor determinant mix) were measured using ImmunoCAP (ThermoFisher Scientific, Uppsala, Sweden). A positive result was defined as a value $\geq 0.35 \mathrm{kU} / \mathrm{L}$. Total IgE levels were measured in 20 of the 22 patients included in the penicillin challenge in patients with previous IgE sensitization to penicillins (ThermoFischer Scientific, Uppsala, Sweden).

Serum elimination half-life $\left(T^{1} / 2\right)$ of s-IgE (pen $G, V, A M P, A X$ ) Determination of the elimination half-life $\left(\mathrm{T}^{1 / 2}\right)$ of $\mathrm{s}$-IgE was calculated using WinNonlin 6.3 (Pharsight, Mountain View, CA, USA). $\mathrm{T}^{1 / 2}$ was calculated by the following equation: $\mathrm{T}^{1 / 2}=\ln [2] / k$, where $k$ is the positive value of the terminal slope of the IgE concentration-time curve. The majority of the patients $(n=20)$ had experienced their initial clinical reaction within one year prior to measurement of the positive s-IgE, three within 5 years and 6 more than five years prior to blood sampling.

\section{Statistics}

Data were analyzed for significance using the Fischer's exact test. Probability values of $<0.05$ were considered statistically significant. The statistical analyses were performed with STATA 13, StataCorp, College Station, Texas, USA.

\section{Results}

The study included 51 cases (48 individual patients, where 3 subjects (all men) were included in both the penicillin challenge in patients with previous IgE sensitization to penicillin and in the estimation of $\mathrm{T}^{1} \frac{1}{2}$ of $\mathrm{s}$-IgE.

Serum elimination half-life ( $T 1 / 2$ ) of $s$-IgE (pen $G, V, A M P, A X$ ) For estimation of $\mathrm{T}^{1} \frac{1}{2}$ of s-IgE, 29 ( 19 women +10 men) patients with three or more consecutively serum measurements of specific IgE to one or more penicillins were 
included. Median level (25-75 percentiles) for s-IgE at inclusion was $4.3 \mathrm{kU} / \mathrm{l}(1.4-18.5)$. Figure 3 shows a scatter-plot of the sequential positive IgE values for all penicillins, where the values are given as percentage of the initial value. A total of 189 measurements of s-IgE were evaluated; 27 initial values of positive specific IgE to pen $\mathrm{V}$ (17 with mono-sensitization), 9 to initial pen G, 10 to initial AMP (2 with mono-sensitization) and 4 to initial AX. In three cases initial specific IgE to all four penicillins were positive. In 8 cases, the specific IgE level was stable over time (up to 55 months). The $\mathrm{T}^{1 / 2}$ for s-IgE between the 26 patients with decreasing s-IgE varied from 1.6 months to 76.4 months. The three patients with stable and increasing IgE-values showed $\mathrm{T}^{1} \frac{1}{2}$ approaching infinity, Figure 4. T1/2 was less than 6 months in $32 \%$ of the patients, $52 \%$ was less than a year and $84 \%$ had a $\mathrm{T}^{1 / 2}$ of less than 3 years.

\section{Penicillin challenge in patients with previous positive specific IgE to penicillins}

Twenty-two patients had a previous positive s-IgE; all were skin test negative to the four penicillins (data not shown). The demographic data, results of specific IgE determination and outcome of their challenge(s) are presented in Table 1. Six of the 22 patients (no. 1-6) had a positive reaction during one of the challenges, three after a single dose (no. 1, 2 and 5), the remaining reacted during (no. 4 and 6) or after (no. 3) the 7-day oral challenge. In 3 of the 6 patients an elevated post-challenge s-IgE was detected. Sixteen patients (no. 7-22) were negative in challenges; in eight of these, (no. 7-14), positive post-challenge s-IgE was observed either after single dose $(n=3)$ or 7 -day oral $(n=5)$ challenge. Post-challenge $\mathrm{s}$-IgE levels showed large individual variation, neither related to initial s-IgE level nor to positive or negative outcome of the challenge, Table 1 . Eleven patients had measurable post-challenge s-IgE, (no. 1, 3, 5, 7-9, 10-14); 3 with positive clinical reaction in challenge ( 2 IR, 1 NIR) and 8 negative (5 IR, $3 \mathrm{NIR}$ ); they were subsequently followed with repeated blood samples as described in Materials and Methods. The post-challenge s-IgE levels declined over time with varying rates, (data not shown). Five patients are currently IgE negative (no. 1, 3, 5, 9, 11), six are still positive (no. 7, 8, 10, 12-14), but specific IgE is declining. The relation between symptoms and signs in case history and during challenge together with postchallenge s-IgE is presented in Table 2. Three patients (no. 7-9) had negative i.v/single dose challenge with penicillin ( $\mathrm{G}$ and $\mathrm{V}$ ) but had positive post-challenge s-IgE to pen $\mathrm{V}$ four weeks later and therefore further challenge was cancelled. In all three subjects pen $\mathrm{V}$ was the culprit drug. Five patients (no. 10-14) showed positive post-challenge s-IgE four weeks after a negative outcome of 7-day oral challenge with pen $\mathrm{V}$. In all 5 cases pen $\mathrm{V}$ was the culprit drug and all had initial positive levels of specific IgE to pen $\mathrm{V}$. In three of the patients (no. 11, 13 and 14), postchallenge s-IgE to other penicillins developed. Eleven patients with either another culprit drug than pen $\mathrm{G}$ or $\mathrm{V}$ (no. 5, 6 (DX), 16-18, 20, 21 (AX)) or positive specific IgE to AMP (no. 5, 6, 9, 14, 15, 19) were also challenged with the culprit drug in case of negative penicillin challenge and negative post-challenge $\mathrm{s}-\mathrm{IgE}(\mathrm{n}=10$, no. 9,14 not challenged, Table 1). Of these, only the two patients with DX as culprit drug (no. 5, 6) were positive in challenge.

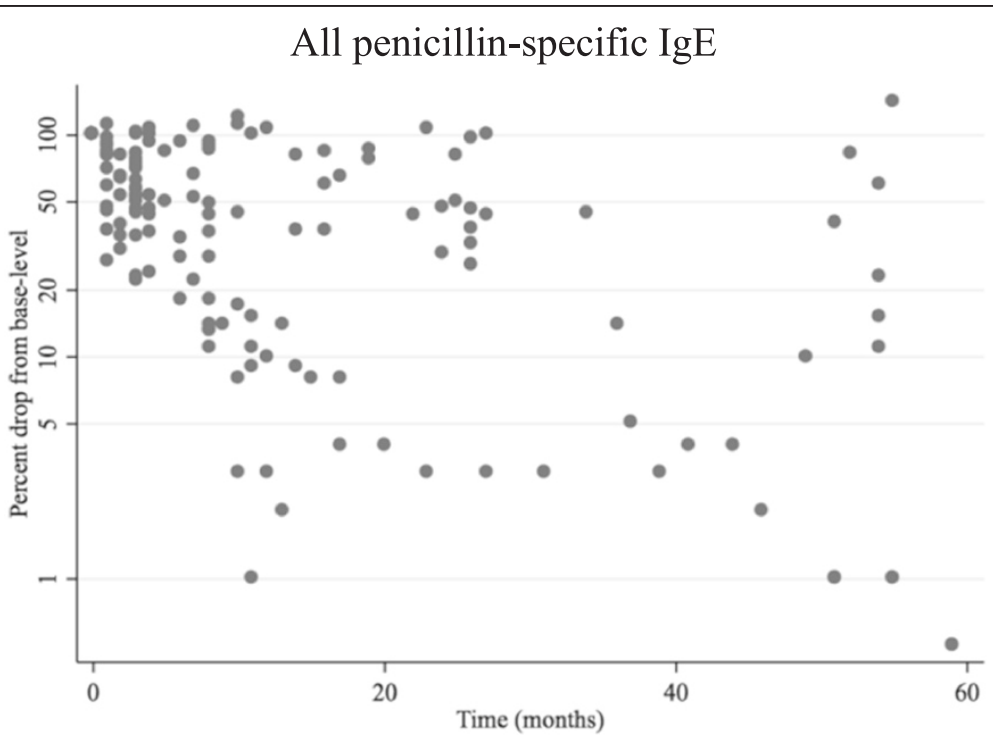

Figure 3 Relative changes in serum concentrations of specific lgE to penicillin presented as the change in amount of specific lgE to penicillin from base line. The level of the patients' first positive s-lgE measurement is set to $100 \%$ on a log-scale. Time of sampling is presented in months from the patients' first positive s-lgE value. All penicillin - specific lgE includes benzylpenicillin, phenoxymethyl penicillin, ampicillin and amoxicillin. 


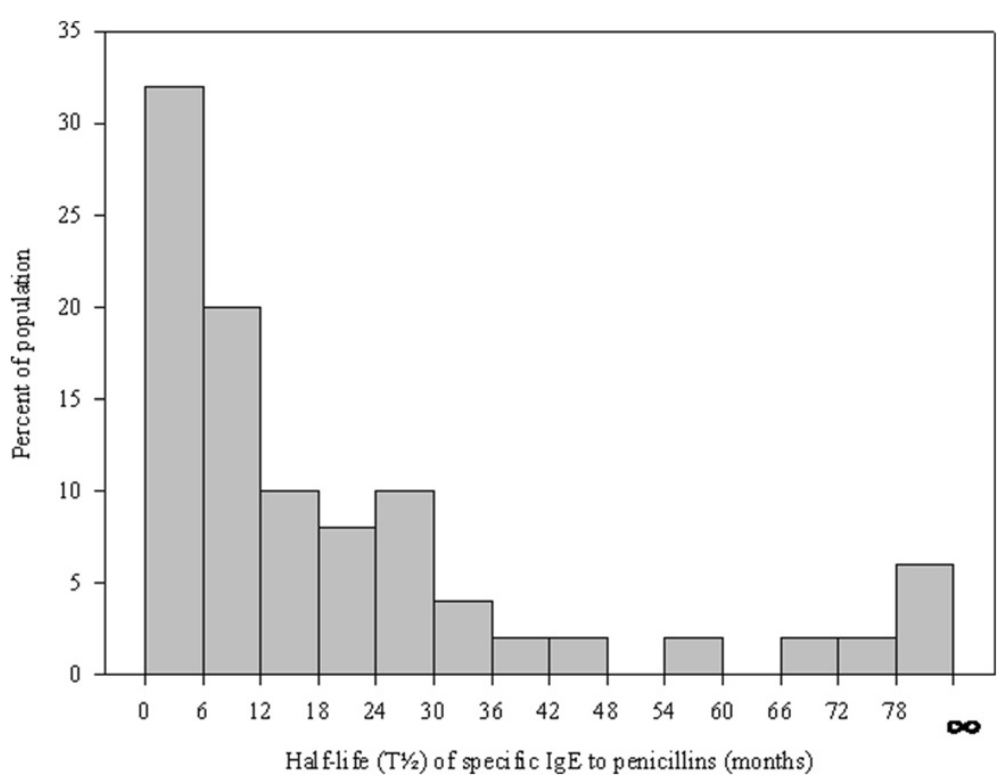

Figure 4 Distribution of the estimated serum half-life $(T 1 / 2)$ of specific $\lg E$ to penicillins. No differences were seen between the four penicillins. $\infty$ : Symbol of infinity, including the 3 cases with stable and increasing IgE-values over time. Specific lgE to penicillins includes benzylpenicillin, phenoxymethyl penicillin, ampicillin and amoxicillin.

Post-challenge level of s-IgE was neither associated with single dose nor 7-day oral challenge outcome, Table 3.

\section{Discussion}

We describe for the first time the outcome of penicillin challenge in patients with a history of penicillin allergy and with previous but not present measurable levels of specific IgE to penicillins. Further, we describe for the first time variations in serum half-life of specific IgE in patients, sensitized to a penicillin.

Serum elimination half-life ( $T 1 / 2)$ of $s-\lg E$ (pen $G, V, A M P, A X$ ) The present study confirms that levels of s-IgE decline over time with different rates in the single patient, neither related to initial level nor to positivity of reaction. Possible underlying mechanisms behind this variable decay is unknown, but in concordance with previous studies having shown that specific IgE antibody levels tend to decrease over time in the absence of new contact with the hapten [9-12]. A decline in IgE antibody responses has previously been described for other allergens such as chlorhexidine [13] and insect venom [14]. The patients in our study had no known exposure to a penicillin after the initial reaction.

$\mathrm{T}^{1 / 2}$ for s-IgE varied from 1.6 to 76.4 months. In approximately half of the patients $\mathrm{T}^{1 / 2}$ was less than a year. Our results thus imply, that a considerable number of penicillin allergic patients will present with a negative s-IgE depending on the time elapsed since their clinical reaction had taken place.

\section{Penicillin challenge in patients with previous IgE sensitization to penicillins}

A total of 29 challenges with $\beta$-lactam antibiotics were performed in 22 patients with previous positive specific IgE to penicillin and negative skin tests. Four different patterns were seen when evaluating the clinical reaction to challenge (positive/negative) and post-challenge boost of s-IgE (yes/no). Eight patients had negative clinical reaction to challenge and negative post-challenge s-IgE, 8 patients had negative challenge, but positive postchallenge specific IgE levels. Three patients had positive clinical reaction to challenge and positive post-challenge s-IgE whereas 3 patients were challenge positive, but had negative post-challenge s-IgE. The six cases with positive challenge should be classified as allergic to penicillin and the 8 cases with negative challenge and negative post-challenge s-IgE should be classified as not being allergic. It is difficult to decide how the 8 patients with negative challenge and subsequent positive postchallenge s-IgE should be classified and they should probably be considered as still being allergic. The appearance of s-IgE after the challenge may be interpreted as a booster reaction. A conclusion can, however, not be drawn without actually challenging the patients once again. Patients with negative s-IgE at the time of investigation and a case history of reaction several years prior to investigation may have been $\mathrm{s}$-IgE positive at the time of reaction. The 22 patients in the present study would have been classified as s-IgE negative if investigated in average 2.7 years after the clinical reaction took place (range $0-8$ years). The frequency of positive outcomes 
Table 1 Demographic data and clinical results of the 22 patients with a history of penicillin allergy and with initial positive serum specific IgE to penicillin

\begin{tabular}{|c|c|c|c|c|c|c|c|c|c|c|c|c|c|c|c|c|c|c|c|c|c|c|c|c|c|c|c|c|}
\hline \multirow{2}{*}{\multicolumn{4}{|c|}{$\begin{array}{c}\text { Patient data* } \\
\begin{array}{l}\text { Age } \\
\text { (years) }\end{array}\end{array}$}} & \multirow{2}{*}{\multicolumn{2}{|c|}{ Primary reaction }} & \multirow{2}{*}{\multicolumn{6}{|c|}{ Initial IgE }} & \multirow{2}{*}{\multicolumn{5}{|c|}{ Iv/single dose challenge }} & \multirow{2}{*}{\multicolumn{4}{|c|}{$\begin{array}{c}\text { Post lgE } \\
\text { (4 w after challenge) }\end{array}$}} & \multirow{2}{*}{\multicolumn{4}{|c|}{ Challenge p.o.7 }} & \multirow{2}{*}{\multicolumn{4}{|c|}{$\begin{array}{c}\text { Post IgE } \\
\text { (4 w after challenge) }\end{array}$}} \\
\hline & & & & & & & & & & & & & & & & & & & & & & & & & & & & \\
\hline No & Sex & $\begin{array}{l}\operatorname{lgE} \\
\text { pos }\end{array}$ & $\begin{array}{l}\lg E \\
\text { neg }\end{array}$ & $\begin{array}{l}\text { Culprit } \\
\text { drug }\end{array}$ & $\begin{array}{l}\text { Type of } \\
\text { reaction }\end{array}$ & Total & $\begin{array}{l}\text { Pen } \\
\text { V }\end{array}$ & $\begin{array}{l}\text { Pen } \\
G\end{array}$ & AMP & $A X$ & MDM & $\begin{array}{l}\text { Pen } \\
\text { G }\end{array}$ & $\begin{array}{l}\text { Pen } \\
\text { V }\end{array}$ & AMP & $A X$ & $\mathrm{DX}$ & $\begin{array}{l}\text { Pen } \\
\text { V }\end{array}$ & $\begin{array}{l}\text { Pen } \\
\text { G }\end{array}$ & AMP & $A X$ & $\begin{array}{l}\text { Pen } \\
\text { V }\end{array}$ & AMP & $A X$ & DX & $\begin{array}{l}\text { Pen } \\
\text { V }\end{array}$ & $\begin{array}{l}\text { Pen } \\
\text { G }\end{array}$ & AMP & $A X$ \\
\hline 1 & $\mathrm{~F}$ & 34 & 35 & Penicillin V & urt (IR) & 53.1 & 0.9 & 0 & 0 & 0 & 0 & Neg & Pos & NT & NT & NT & 2.8 & 0 & 0 & 0 & 1 & NT & NT & NT & NT & NT & NT & NT \\
\hline 2 & M & 65 & 69 & Penicillin V & urt (NIR) & 248.6 & 0.4 & 0 & 0 & 0 & 0 & Neg & Pos & NT & NT & NT & 0 & 0 & 0 & 0 & 1 & NT & NT & NT & NT & NT & NT & NT \\
\hline 3 & M & 38 & 38 & Penicillin V & $\begin{array}{l}\text { urt+angio } \\
\text { (NIR) }\end{array}$ & 101.5 & 0.5 & 0 & 0 & 0 & 0 & Neg & Neg & NT & NT & NT & 0 & 0 & 0 & 0 & Pos & NT & NT & NT & 0.4 & 0 & 0 & 0 \\
\hline 4 & M & 18 & 19 & Penicillin V & urt (NIR) & 54.1 & 0.4 & 0 & 0 & 0 & 0 & Neg & Neg & NT & NT & NT & 0 & 0 & 0 & 0 & Pos & NT & NT & NT & 0 & 0 & 0 & 0 \\
\hline 5 & M & 56 & 57 & Dicloxacillin & $\begin{array}{l}\text { urt+angio } \\
\text { (IR) }\end{array}$ & 271.7 & 0.5 & 0.4 & 0.8 & 0 & 0 & NT & NT & NT & NT & Pos & 0.4 & 0 & 0.4 & 0 & 1 & NT & NT & NT & NT & NT & NT & NT \\
\hline 6 & M & 77 & 81 & Dicloxacillin & rash (NIR) & 1773.0 & 0 & 0 & 0.7 & 0 & 0 & Neg & Neg & NT & NT & Neg & 0 & 0 & 0 & 0 & Neg & NT & NT & Pos & 0 & 0 & 0 & 0 \\
\hline 7 & F & 30 & 34 & Penicillin V & urt (IR) & 4.2 & 0.9 & 0.8 & 0 & 0 & 0 & Neg & Neg & NT & NT & NT & 1.2 & 0.5 & 0 & 0 & $\backslash$ & NT & NT & NT & NT & NT & NT & NT \\
\hline 8 & M & 30 & 33 & Penicillin V & urt (IR) & 26.8 & 0.4 & 0 & 0 & 0 & 0 & Neg & Neg & NT & NT & NT & 0.8 & 0 & 0 & 0 & 1 & NT & NT & NT & NT & NT & NT & NT \\
\hline 9 & M & 51 & 56 & Penicillin V & rash (NIR) & 580.7 & 0 & 0 & 0.4 & 0 & 0 & Neg & Neg & NT & NT & NT & 0.5 & 0.7 & 0 & 0 & 1 & NT & NT & NT & NT & NT & NT & NT \\
\hline 10 & M & 43 & 43 & Penicillin V & rash $(\mathrm{IR})$ & 10.2 & 0.4 & 0 & 0 & 0 & 0 & Neg & Neg & NT & NT & NT & 0 & 0 & 0 & 0 & Neg & NT & NT & NT & $>100$ & 0 & 0 & 0 \\
\hline 11 & M & 58 & 58 & Penicillin V & urt (IR) & 142.7 & 0.4 & 0 & 0 & 0 & 0 & Neg & Neg & NT & NT & NT & 0 & 0 & 0 & 0 & Neg & NT & NT & NT & 1.5 & 0.4 & 0 & 0 \\
\hline 12 & $\mathrm{~F}$ & 48 & 49 & Penicillin V & $\begin{array}{l}\text { urt+angio } \\
(\mathrm{IR})\end{array}$ & 38.3 & 0.8 & 0 & 0 & 0 & 2.3 & Neg & Neg & NT & NT & NT & 0 & 0 & 0 & 0 & Neg & NT & NT & NT & 3.9 & 0 & 0 & 0 \\
\hline 13 & M & 51 & 55 & Penicillin V & rash (NIR) & 363.7 & 0.4 & 0 & 0 & 0 & 0 & Neg & Neg & NT & NT & NT & NT & NT & NT & NT & Neg & NT & NT & NT & 4.3 & 0.4 & 0.4 & 0 \\
\hline 14 & $\mathrm{~F}$ & 46 & 49 & Penicillin V & $\begin{array}{l}\text { urt+angio } \\
\text { (NIR) }\end{array}$ & 4.3 & 3.4 & 1.4 & 0.5 & 0 & 0 & Neg & Neg & NT & NT & NT & 0 & 0 & 0 & 0 & Neg & NT & NT & NT & 9.8 & 2.8 & 2.9 & 1.3 \\
\hline 15 & $\mathrm{~F}$ & 34 & 38 & Penicillin V & rash (NIR) & 491.2 & 0 & 0 & 0.9 & 0 & 0 & Neg & Neg & Neg & NT & NT & 0 & 0 & 0 & 0 & Neg & Neg & NT & NT & 0 & 0 & 0 & 0 \\
\hline 16 & $\mathrm{~F}$ & 63 & 67 & Amoxicillin & rash (NIR) & 107.3 & 0.4 & 0 & 0 & 0 & 0 & Neg & Neg & NT & Neg & NT & 0 & 0 & 0 & 0 & Neg & NT & Neg & NT & 0 & 0 & 0 & 0 \\
\hline 17 & $M$ & 60 & 61 & Amoxicillin & rash (NIR) & 698.1 & 3.2 & 0 & 0 & 0 & 0 & Neg & Neg & NT & NT & NT & 0 & 0 & 0 & 0 & Neg & NT & Neg & NT & 0 & 0 & 0 & 0 \\
\hline 18 & $\mathrm{~F}$ & 60 & 67 & Amoxicillin & urt (NIR) & NT & 0.5 & 0 & 0 & 0 & 0 & Neg & Neg & NT & Neg & NT & 0 & 0 & 0 & 0 & NT & NT & Neg & NT & 0 & 0 & 0 & 0 \\
\hline 19 & M & 52 & 52 & Penicillin V & rash (NIR) & NT & 0 & 0 & 0.7 & 0 & 0 & Neg & Neg & Neg & NT & NT & NT & NT & NT & NT & Neg & Neg & NT & NT & NT & NT & NT & NT \\
\hline 20 & $\mathrm{~F}$ & 56 & 61 & Amoxicillin & rash (NIR) & NT & 1.1 & 1.0 & 0 & 0 & 0 & Neg & Neg & NT & Neg & NT & NT & NT & NT & NT & Neg & NT & Neg & NT & NT & NT & NT & NT \\
\hline 21 & $\mathrm{~F}$ & 58 & 59 & Amoxicillin & rash (NIR) & NT & 0.4 & 0 & 0 & 0 & 0 & Neg & Neg & NT & Neg & NT & NT & NT & NT & NT & Neg & NT & Neg & NT & NT & NT & NT & NT \\
\hline 22 & $\mathrm{~F}$ & 35 & 43 & Penicillin V & urt (NIR) & 355.8 & 0.4 & 0 & 0 & 0 & 0 & Neg & Neg & NT & NT & NT & NT & NT & NT & NT & Neg & NT & NT & NT & NT & NT & NT & NT \\
\hline
\end{tabular}

"Patient data are illustrating the patients' age at the time of respective IgE positive and IgE negative results.

positive i.v: male, urt: urticarial, angio: angioedema, p.o.7: 7-day oral challenge, pos: positive challenge, neg: negative challenge, NT: 
Table 2 Relation between clinical reaction (case history) and reaction at penicillin challenge, eliciting penicillin, challenge type and post-challenge levels of $s-\lg E$

\begin{tabular}{cccccc}
\hline & \multicolumn{2}{c}{ Type of reaction } & & Type of challenge & \multicolumn{2}{c}{ Post-challenge s-IgE } \\
Patient no. & Case history & Challenge & & Positive & 4 weeks later \\
\cline { 2 - 4 } 1 & Urticaria (IR) & Urticaria (IR) & & Single p.o Pen V & Positive \\
2 & Urticaria (NIR) & Maculopapular rash (NIR) & Single p.o Pen V & Negative \\
3 & Urticaria/angioedema (NIR) & Urticaria (NIR) & P.o.7 Pen V & Positive \\
4 & Urticaria (NIR) & Urticaria (NIR) & P.o.7 Pen V & Negative \\
5 & Urticaria/angioedema (IR) & Urticaria (IR) & P.o.7 DX & Positive \\
6 & Urticaria (NIR) & Maculopapular rash (NIR) & P.o.7 DX & Negative \\
\hline
\end{tabular}

Bold text indicate concordance between type of reactions eliciting primary reaction and challenge. In the three cases of positive post-challenge s-IgE (no. $1,3,5$ ) concordance was found.

In all cases, the culprit drug and the drug eliciting the clinical reaction during challenge were identical (see Table 1).

IR: immediate reaction, NIR: nonimmediate reaction, s-IgE: serum specific IgE to penicillin, p.o.7: 7-day oral challenge, p.o: per oral, Pen V: phenoxymethylpenicillin, DX: dicloxacillin.

of penicillin challenge in the present study $(6 / 29$ challenges $-20.7 \%$ ) was similar to the findings in our previous study [2], where all patients challenged had a positive history but negative specific IgE to penicillin. Out of 291 challenges 50 had a positive reaction (17.2\%) despite that the patients in the present study had previous IgE sensitization.

\section{Positive and negative predictive value of serum specific IgE in patients with a history of penicillin allergy and negative skin tests}

Several studies have evaluated $[7,15-20]$ the positive and negative predictive value of $\mathrm{s}$-IgE in patients with a positive history of penicillin allergy but negative skin tests by performing oral/i.v. challenge test. Silva et al. [7] found that only 2 of 7 patients with positive s-IgE had a positive reaction to a challenge test, both were IR with cutaneous symptoms, indicating that a positive s-IgE in patients with a history of penicillin hypersensitivity and negative skin test is not sufficient for confirming the diagnosis. In the same study 2 of 15 patients with negative s-IgE had a positive reaction after challenge, giving a positive predictive value (PPV) of $29 \%(2 / 7)$ and a negative predictive value (NPV) of $89 \%(13 / 15)$. In another study Macy et al. [15] challenged four patients with positive s-IgE (skin test negative), all with negative outcome. Aberer et al. [16-18] stated that detection of IgE antibodies to penicillin does prove sensitization, but not the existence of a clinical allergy. Torres et al. [19] who studied 40 patients with positive s-IgE and negative skin test, found however that 24 patients had a positive IR (immediate reaction) to penicillin during challenge. Blanca et al. [20] challenged $3 \mathrm{~s}$-IgE positive, skin test negative patients with a positive reaction in two only. Both authors describe a high predictive value of a positive s-IgE indicating that challenging patients with current s-IgE will increase the possibility of a positive outcome. This in contrast to our findings, when challenging presently negative but previously IgE positive patients.

\section{Immediate reactions (IR)/Nonimmediate reactions (NIR)}

Measurement of s-IgE is recommended in patients with a history of an immediate reaction (IR) [3]. Due to the low predictive value of the patient history, [2] we recommend both IR and NIR to be evaluated using the same diagnostic work-up. In this study all 7 patients with IR in case history ended up with positive post-challenge sIgE, but this was also the case in 4 of the 15 patients with NIR in case history (Table 1). Only 2 respective 1 were challenge positive. S-IgE to penicillin should therefore be

Table 3 Specific IgE level (positive/negative) compared to outcome of penicillin challenge (positive/negative)

\begin{tabular}{|c|c|c|c|c|c|c|c|c|c|}
\hline & & \multicolumn{2}{|c|}{ I.v/single p.o (pen V,G) $(n=21)^{* *}$} & \multicolumn{2}{|c|}{ P.o.7 (pen V) $(n=15)^{*}$} & \multicolumn{2}{|c|}{ Single p.o (other) $(n=8)^{*}$} & \multicolumn{2}{|c|}{ P.o.7 (other) $(n=8)^{*}$} \\
\hline & & $\operatorname{Pos}(n=2)$ & $\operatorname{Neg}(n=19)$ & $\operatorname{Pos}(n=2)$ & $\operatorname{Neg}(n=13)$ & $\operatorname{Pos}(n=1)$ & $\operatorname{Neg}(n=7)$ & $\operatorname{Pos}(n=1)$ & $\operatorname{Neg}(n=7)$ \\
\hline \multirow{3}{*}{$\begin{array}{l}\text { Post-challenge } \\
\text { s-lgE }\end{array}$} & Pos $(n=11)$ & 1 & 3 & 1 & 5 & $1^{* *}$ & 0 & 0 & 0 \\
\hline & Neg $(n=25)$ & 1 & $11^{*}$ & 1 & 4 & 0 & 4 & 1 & $4^{*}$ \\
\hline & NT $(n=12)$ & 0 & 5 & 0 & 4 & 0 & 3 & 0 & 3 \\
\hline
\end{tabular}

A total of 21 patients were challenged with i.v/single p.o pen $\mathrm{V}$, followed by p.o.7 pen $\mathrm{V}$ in 15 patients. **Patient no. 5 (Table 1 ) was only challenged with culprit drug (DX).

Nine patients also challenged with other drug (either their culprit drug or because of previous positive specific lgE to AMP or AX). In 8 cases initiated by single p.o, and in 1 case* (no. 17, Table 1) initiated by challenge with pen $\mathrm{G}$ and V (i.v/single p.o), followed by challenge with AX (culprit drug) in p.o.7 (not preceded by single p.o AX). No. 17, Table 1 had not performed p.o.7 with pen $\mathrm{V}$.

s-IgE: serum specific IgE to penicillin, I.v: intravenous, Single p.o: single dose oral challenge, P.o.7: 7-day oral challenge, Pen V: phenoxymethylpenicillin, Pen G: benzylpenicillin, DX: dicloxacillin, AX: amocxicillin, Pos: positive, Neg: negative, NT: not tested. 
measured in all patients with suspected penicillin allergy also since many patients are unable to give a detailed case history regarding time interval between dosage and reaction $[21,22]$ and further due to the fact that s-IgE both can be positive in challenge proven immediate as well as nonimmediate responders. Ideally, due to the short $\mathrm{T}^{1} 12$ in many patients, s-IgE should be measured within 6 months after a reaction to penicillin occurred.

\section{The significance of positive specific IgE to penicillin}

In a study from 2013 Johansson et al. [23] indicated that false-positive penicillin immunoassays constitute an unnoticed, but rather common problem. They found that $26 \%$ of patients with a suspected IgE-mediated reaction to penicillin and a positive ImmunoCAP might have specific IgE antibodies to the clinically irrelevant phenylethylamine with a benzyl group (PEA) instead. This may be an argument for measurement of PEA antibodies and for the confirmation of an allergy by clinical tests. Three studies [23-25] have stated that very low levels of allergen s-IgE should be evaluated with caution when serum level of total IgE is above $500 \mathrm{kU} / \mathrm{l}$ and that the specificity of tests for anti- $\beta$-lactam IgE antibodies declines progressively with increase of total serum IgE. Total serum IgE values were measured in 18 patients (Table 1). Only 3 had total IgE values above $500 \mathrm{kU} / \mathrm{l}$ (8 exceeding $150 \mathrm{kU} / \mathrm{l})$. The patient with the highest level of total serum $\operatorname{IgE}(1773 \mathrm{kU} / \mathrm{l})$ was challenge positive, whereas the others were negative, supporting the theory that elevated level of total IgE may interfere with measurements of specific IgE to penicillins. No significant difference between the patients with high and normal total IgE regarding atopy was found $(\mathrm{p}>0.05)$, data not shown. Kraft et al. [12] have also shown that patients with acute allergic reactions to penicillin have high levels of total serum IgE and that it subsequent decreases in tune with the decline of specific IgE to penicillins.

\section{Conclusion}

An IgE response to penicillin is often a transient phenomenon. In this study approximately half of the patients had a serum half-life of specific IgE to penicillin of less than a year, suggesting that the majority of patients with IgE sensitization at the time of reaction have converted to IgE negativity if measured more than a year after the incident took place. This is supported by the finding that we found the same frequency of positive outcomes during penicillin challenge in the present study compared to our previous study, where all patients (with a case history of penicillin allergy) challenged never had had demonstrable IgE to penicillin [2] in contrast to the present study where patients had previous IgE sensitization. Regarding the challenges with penicillin in patients with a previous but not present positive s-IgE, a negative sIgE was not associated with tolerance in 63.6\% of the tested patients and even a tolerated readministration of penicillins (also during iatrogenic diagnostic workup) may lead to boostering of sIgE with unknown significance, but possibly reoccurrence of penicillin allergy. Further the more indistinct outcomes in the patients with negative challenge but positive post-challenge s-IgE, no firm conclusion can be drawn without challenging the patients once again. Our results thus suggest that a negative outcome of the 7-day oral challenge should be followed by measurement of specific IgE 4 weeks later, and only if this proved negative, the patient should be classified as tolerant to penicillin.

\section{Abbreviations \\ AMP: Ampicillin; AX: Amoxicillin; b.i.d: Twice a day; B-lactam: Betalactam antibiotic; DX: Dicloxacillin; ICT: Intracutaneous; IE: International units; IgE: Immunoglobulin E; ImmunoCap: Specific immunoglobulin E blood test (Pharmacia Diagnostics AB, Uppsala, Sweden); IR: Immediate reaction; i.v: Intravenous; NIR: Nonimmediate reaction; NPV: Negative predictive value; P: p-value: Significance level; PEA: Phenylethylamine with a benzyl group; Pen G: Benzylpenicillin; Pen V: Phenoxymethylpenicillin; p.o.7: 7-day oral challenge; PPV: Positive predictive value; s-IgE: Serum specific immunoglobulin E to penicillins; SPT: Standard skin prick test; $T 1 \frac{12}{2}$ : Serum elimination half-life; t.i.d: Three times a day.}

\section{Competing interests}

The authors declare that they have no competing interests.

\section{Authors' contributions}

All listed authors have met the requirements approved by the International Committee of Medical Journal Editors. JH contributed to conception and design of study and interpretation and discussion of the results. $\mathrm{JH}$ also performed the data analysis and drafted the manuscript. CG, PSS, RD and CBJ contributed to conception and design of study and interpretation and discussion of the results. TBS performed the analysis regarding the estimated serum half-life of specific IgE to penicillins. All the authors approved the final version.

\section{Author details}

'Department of Dermatology and Allergy Centre, Odense University Hospital, Sdr. Boulevard 29, DK-5000 Odense C, Denmark. ${ }^{2}$ Clinical Pharmacology, Institute of University of Southern Denmark, Odense C, Denmark.

Received: 19 June 2014 Accepted: 18 September 2014 Published: 29 October 2014

\section{References}

1. Hjortlund J, Mortz CG, Skov PS, Eller E, Poulsen JM, Borch JE, Bindslev Jensen C: One-week oral challenge with penicillin in diagnosis of penicillin allergy. Acta Derm Venereol 2012, 92:307-312.

2. Hjortlund J, Mortz CG, Skov PS, Bindslev-Jensen C: Diagnosis of penicillin allergy revisited: the value of case history, skin testing, specific IgE and prolonged challenge. Allergy 2013, 68:1057-1064.

3. Torres MJ, Blanca M, Fernandez J, Romano A, Weck A, Aberer W, Brockow K, Pichler WJ, Demoly P: Diagnosis of immediate allergic reactions to beta-lactam antibiotics. Allergy 2003, 58:961-972.

4. Romano A, Blanca M, Torres MJ, Bircher A, Aberer W, Brockow K, Pichler W, Demoly P: Diagnosis of nonimmediate reactions to beta-lactam antibiotics. Allergy 2004, 59:1153-1160.

5. Blanca M, Romano A, Torres MJ, Fernandez J, Mayorga C, Rodriguez J, Demoly P, Bousquet PJ, Merk HF, Sanz ML, Ott H, Atanaskovic-Markovic M: Update on the evaluation of hypersensitivity reactions to betalactams. Allergy 2009, 64:183-193.

6. Demoly P, Adkinson NF, Brockow K, Castells M, Chiriac AM, Greenberger PA, Khan DA, Lang DM, Park HS, Pichler W, Sanchez-Borges M, Shiohara T, 
Thong BY: International consensus on drug allergy. Allergy 2014, 69:420-437.

7. Silva R, Cruz L, Botelho C, Castro E, Cadinha S, Castel-Branco MG, Rodrigues J: Immediate hypersensitivity to penicillins with negative skin tests-the value of specific IgE. Eur Ann Allergy Clin Immunol 2009, 41:117-119.

8. Fontaine C, Mayorga C, Bousquet PJ, Arnoux B, Torres MJ, Blanca M, Demoly P. Relevance of the determination of serum-specific lgE antibodies in the diagnosis of immediate beta-lactam allergy. Allergy 2007, 62:47-52

9. Mayorga C, Sanz ML, Gamboa PM, Garcia BE, Caballero MT, Garcia JM, Labrador M, Lahoz C, Longo AN, Lopez HM, Martinez QJ, Monteseirin FJ: In vitro diagnosis of immediate allergic reactions to drugs: an update. J Investig Allergol Clin Immunol 2010, 20:103-109.

10. Fernandez T, Torres MJ, Pena R, Fuentes MS, Robles S, Mayorga C, Blanca M: Decrease of selective immunoglobulin $\mathrm{E}$ response to amoxicillin despite repeated administration of benzylpenicillin and penicillin V. Clin Exp Allergy 2005, 35:1645-1650.

11. Fernandez TD, Torres MJ, Blanca-Lopez N, Rodriguez-Bada JL, Gomez E, Canto G, Mayorga C, Blanca M: Negativization rates of IgE radioimmunoassay and basophil activation test in immediate reactions to penicillins. Allergy 2009, 64:242-248.

12. Kraft D, Roth $A$, Mischer $P$, Pichler $H$, Ebner $H$ : Specific and total serum IgE measurements in the diagnosis of penicillin allergy. A long term follow-up study. Clin allergy 1977, 7:21-28.

13. Garvey LH, Kroigaard M, Poulsen LK, Skov PS, Mosbech H, Venemalm L, Degerbeck F, Husum B: IgE-mediated allergy to chlorhexidine. J Allergy Clin Immunol 2007, 120:409-415.

14. Mosbech H, Christensen J, Dirksen A, Soborg M: Insect allergy. Predictive value of diagnostic tests: a three-year follow-up study. Clin allergy 1986, 16:433-440.

15. Macy E, Goldberg B, Poon $\mathrm{KY}$ : Use of commercial anti-penicillin IgE fluorometric enzyme immunoassays to diagnose penicillin allergy. Ann Allergy Asthma Immunol 2010, 105:136-141.

16. Geyer E, Derhaschnig J, Aberer W: IgE response to penicillin. Allergy 1997, 52:671.

17. Aberer $W$, Zidarn $M$, Kranke B: IgE antibodies to penicillin are indicative for but not conclusive proof of penicillin allergy. Br J Dermatol 2006, 154:1209-1210.

18. Aberer W, Kranke B, Hager A, Wick G: In vitro allergy testing needs better standardization-test results from different laboratories lack comparability mostly due to missing effective standards. Int Arch Allergy Immunol 1995, 108:82-88.

19. Torres MJ, Mayorga C, Cornejo-Garcia JA, Romano A, Blanca M: IgE antibodies to penicillin in skin test negative patients. Allergy 2002, 57:965.

20. Blanca M, Mayorga C, Torres MJ, Reche M, Moya MC, Rodriguez UL, Romano A, Juarez C: Clinical evaluation of pharmacia CAP system RAST FEIA amoxicilloyl and benzylpenicilloyl in patients with penicillin allergy. Allergy 2001, 56:862-870.

21. Demoly P, Bousquet J: Drug allergy diagnosis work up. Allergy 2002, 57:37-40.

22. Schnyder B, Pichler W: Nonimmediate drug allergy: diagnostic benefit of skin testing and practical approach. J Allergy Clin Immunol 2012, 129:1170-1171.

23. Johansson SG, Adedoyin J, Van HM, Gronneberg R, Nopp A: False-positive penicillin immunoassay: an unnoticed common problem. J Allergy Clin Immunol 2013, 132:235-237.

24. Kopac P, Zidarn M, Kosnik M: Epidemiology of hypersensitivity reactions to penicillin in Slovenia. Acta DermatovenerolAlp Panonica Adriat 2012. 21:65-67.

25. Zidarn M, Silar M, Vegnuti M, Korosec P, Kosnik M: The specificity of tests for anti-beta-lactam IgE antibodies declines progressively with increase of total serum IgE. Wien Klin Wochenschr 2009, 121:353-356.

doi:10.1186/2045-7022-4-34

Cite this article as: Hjortlund et al:: Positive serum specific IgE has a short half-life in patients with penicillin allergy and reversal does not always indicate tolerance. Clinical and Translational Allergy 2014 4:34.

\section{Submit your next manuscript to BioMed Central and take full advantage of:}

- Convenient online submission

- Thorough peer review

- No space constraints or color figure charges

- Immediate publication on acceptance

- Inclusion in PubMed, CAS, Scopus and Google Scholar

- Research which is freely available for redistribution

Submit your manuscript at www.biomedcentral.com/submit
Ciomed Central 